\title{
Phase-dependent heat transport through magnetic Josephson tunnel junctions
}

\author{
F. S. Bergeret ${ }^{1,2,3, *}$ and F. Giazotto ${ }^{4, \dagger}$ \\ ${ }^{1}$ Centro de Física de Materiales (CFM-MPC), Centro Mixto CSIC-UPV/EHU, Manuel de Lardizabal 4, E-20018 San Sebastián, Spain \\ ${ }^{2}$ Donostia International Physics Center (DIPC), Manuel de Lardizabal 5, E-20018 San Sebastián, Spain \\ ${ }^{3}$ Institut für Physik, Carl von Ossietzky Universität, D-26111 Oldenburg, Germany \\ ${ }^{4}$ NEST, Instituto Nanoscienze-CNR and Scuola Normale Superiore, I-56127 Pisa, Italy
}

(Received 27 May 2013; published 19 July 2013)

\begin{abstract}
We present an exhaustive study of the coherent heat transport through superconductor-ferromagnet (SF) Josephson junctions including a spin-filter $\left(\mathrm{I}_{\mathrm{sf}}\right)$ tunneling barrier. By using the quasiclassical Keldysh Green's function technique we derive a general expression for the heat current flowing through a SF-I sf $_{-}-\mathrm{FS}$ junction and analyze the dependence of the thermal conductance on the spin-filter efficiency, the phase difference between the superconductors and the magnetization direction of the ferromagnetic layers. In the case of noncollinear magnetizations we show explicitly the contributions to the heat current stemming from the singlet and triplet components of the superconducting condensate. We also demonstrate that the magnetothermal resistance ratio of a SF- $\mathrm{I}_{\mathrm{sf}}-\mathrm{FS}$ heat valve can be increased by the spin-filter effect under suitable conditions.
\end{abstract}

DOI: 10.1103/PhysRevB.88.014515

PACS number(s): 74.50.+r, 74.25.F-

\section{INTRODUCTION}

Two fields have been attracting increasing attention among several research groups recently: spintronics with superconductors $^{1-4}$ and coherent caloritronics. ${ }^{5-21}$ Both fields exploit phase-dependent phenomena, which are key characteristics of superconducting circuits. On the one hand, superconducting spintronics is emerging as a possible technology from the discovery of spin-polarized supercurrents ${ }^{3}$ in superconductor-ferromagnet (SF) hybrid nanostructures. Such supercurrents are due to the existence of triplet superconducting correlations created by magnetic inhomogeneities. ${ }^{22}$ Once generated, triplet correlations can penetrate over long distances into ferromagnets as observed in experiments on SFS Josephson junctions. ${ }^{23-26}$ These experiments suggest the possibility of using SF hybrids in spintronic circuits with the aim of lowering the dissipation. ${ }^{4}$ On the other hand, the study of heat transport in nanoscale devices, i.e., caloritronics, also attracts the attention of researchers working on nanodevies ${ }^{27-29}$ containing, for example, normal metal, ferromagnets ${ }^{30,31}$ and superconductors. ${ }^{32,33}$ Of particular interest is the recent experimental control of the heat current flowing through a Josephson junction by tuning the macroscopic phase difference between two superconducting reservoirs, ${ }^{5,34,35}$ as predicted in several theoretical works. ${ }^{36-40}$

The interplay between superconductivity and ferromagnetism in the context of heat transport has been used to describe a phase-tunable heat-valve in a recent theoretical work. ${ }^{41}$ The valve is a FS-I-SF Josephson junction (I denotes a nonmagnetic tunneling barrier) and its operating principle is based on both phase coherence and spin-dependent transport. Moreover, it is well known that in junctions containing SF elements both singlet and triplet pair correlations are generated and contribute to the Josephson (charge) current and, as we will prove below, to the phase-dependent part of the heat current. If a spin filter with a large efficiency is used as a tunneling barrier, the singlet contribution to the Josephson current is suppressed and a highly spin-polarized supercurrent can be achieved in a $\mathrm{SF}-\mathrm{I}_{\mathrm{sf}}$-FS junction provided that the magnetizations are noncollinear ${ }^{42}\left(\mathrm{I}_{\mathrm{sf}}\right.$ denotes the spin-filter tunneling barrier). As we shall show in the present work, this also applies for the phase-coherent part of the heat current flowing through a $\mathrm{SF}-\mathrm{I}_{\mathrm{sf}}-\mathrm{FS}$ junction.

The spin-filter effect has been intensively studied in europium chalcogenides tunneling barriers. ${ }^{43-46}$ This type of barrier possesses very large spin-filter efficiencies (typically larger than 95\%) and, therefore, they are ideal candidates for the creation of spin-polarized currents. In tunnel junctions made of superconducting electrodes and spin-filter barriers, measurements of the tunneling conductance have revealed that the interaction between conducting electrons in the leads and the localized magnetic moments of the barrier lead to a Zeeman splitting in the density of states of the superconducting electrodes, ${ }^{43,47}$ as theoretically expected. ${ }^{48}$ An experiment performed on $\mathrm{NbN}-\mathrm{GdN}-\mathrm{NbN}$ junctions has shown that the temperature dependence of the Josephson current flowing through a GdN barrier (with a spin-filter efficiency of $~ 75 \%$ ) clearly deviates from that expected in conventional S-I-S junctions, ${ }^{49}$ thus suggesting an interplay between magnetism of the barrier and superconducting condensate of the electrodes, as described recently in a theoretical work. ${ }^{42}$

In the present work we combine ideas from SF hybrid structures and caloritronics studies in order to analyze the phase-dependent heat transport through such structures. We extend the model proposed in Ref. 50 for the heat transport through $\mathrm{S}-\mathrm{I}_{\mathrm{sf}}-\mathrm{S}$ and $\mathrm{FS}-\mathrm{I}_{\mathrm{sf}}-\mathrm{SF}$ junctions and derive compact expressions for the thermal conductance. With the help of our model we are able to study in detail the dependence of the heat conductance on the spin-filter efficiency, the superconducting phase and the relative angles between the magnetization of the ferromagnetic layers. In analogy to the charge supercurrent we shall demonstrate that the phase-dependent part of the heat current consists of two contributions stemming from singlet and triplet pair correlations, respectively. Moreover, as for the charge transport studied in Ref. 42, the spin-filter effect suppresses the singlet contribution to thermal transport leading to spin-polarized heat currents. Finally, we show how the spin-filter barriers can be used for the enhancement of the magnetothermal resistance of Josephson heat valves as those recently proposed in Ref. 41. 
The paper is organized as follows. In Sec. II we derive a general expression describing the heat current flowing through a generic spin-filter junction. With the help of this expression, in Sec. III we first analyze the heat conductance through a $\mathrm{S}-\mathrm{I}_{\mathrm{sf}}-\mathrm{S}$ junction as the one used in the experiments. ${ }^{43,49} \mathrm{We}$ demonstrate that while for a zero-phase difference between the superconductors the thermal conductance increases by increasing the spin-filter efficiency, the opposite regime is achieved if the phase difference $\phi$ equals to $\pi$. This behavior holds in the presence of a Zeeman splitting in the superconductors and also if we neglect this field. We also show that for a large spin-filter efficiency of the barrier the maximum value of the thermal conductance depends nonmonotonically on the amplitude of the Zeeman splitting. In Sec. III B we consider a triplet Josephson junction consisting of a F-S- $\mathrm{I}_{\mathrm{sf}}-\mathrm{SF}$ structure, for which the magnetization direction of the outer $F$ layers can point in arbitrary direction with respect to the spin quantization axis determined by the magnetization of the $I_{s f}$ barrier. We explicitly show the contributions of the singlet and triplet part of the condensate to the heat conductance. In Sec. III C we discuss the ferromagnetic Josephson thermal valve and show that the magnetothermal resistance ratio in the structure can reach values as large as $10^{6}-10^{8}$ at low temperature depending on the macroscopic phase and on the spin-filter efficiency of the barrier. Finally, we summarize our results in Sec. IV.

\section{MODEL}

We consider the generic Josephson junction sketched in Fig. 1(a). It consists of two SF electrodes tunnel coupled by a spin-filter barrier $I_{s f}$. The thin $F$ layers may model the

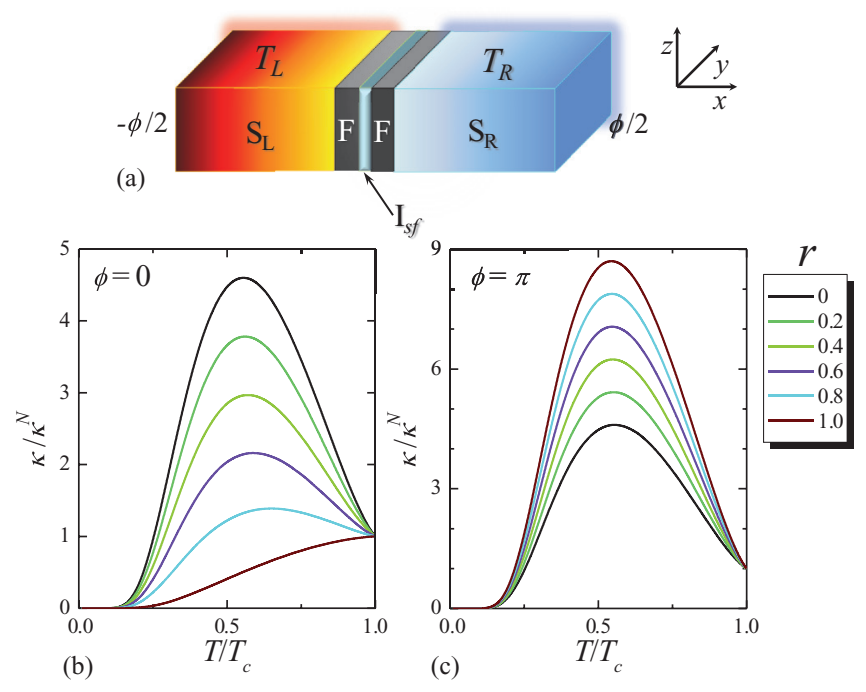

FIG. 1. (Color online) (a) Sketch of the generic SF-I $\mathrm{I}_{\mathrm{sf}}-\mathrm{FS}$ Josephson tunnel junction. It consists of two identical SF layers coupled by a spin-filter barrier $\mathrm{I}_{\mathrm{sf}}$. $T_{L}$ and $T_{R}$ denotes the temperatures of the superconductors whereas $\phi$ is the macroscopic quantum phase difference over the junction. (b) Thermal conductance $\kappa$ vs temperature $T$ calculated for different values of $r$ at $\phi=0$ in the absence of Zeeman splitting in the superconducting electrodes. (c) The same quantity as in panel (b) calculated for $\phi=\pi . \kappa^{N}$ denotes the thermal conductance in the normal state while $T_{c}$ is the superconducting critical temperature. effective exchange field induced in the $\mathrm{S}$ electrodes due to the presence of the magnetic barrier. ${ }^{42}$ This model is accurate if one assumes that the $\mathrm{F}$ and $\mathrm{S}$ layers are in good electric contact and their thicknesses are small enough. ${ }^{51}$ The junction is phase and temperature biased. The phase difference between the left $(\mathrm{L})$ and right $(\mathrm{R})$ electrode is denoted by $\phi$, while their temperatures are kept constant, at $T_{L}$ and $T_{R}$, respectively. In order to describe the electronic transport in the junction we introduce the quasiclassical Green's functions (GFs) in the $\mathrm{L}$ and $\mathrm{R}$ electrodes, which are $8 \times 8$ matrices in the Nambu-spin-Keldysh space

$$
\mathbf{G}_{\mathrm{R}(\mathrm{L})}=\left(\begin{array}{ll}
\check{G}_{\mathrm{R}(\mathrm{L})}^{R} & \check{G}_{\mathrm{R}(\mathrm{L})}^{K} \\
0 & \check{G}_{\mathrm{R}(\mathrm{L})}^{A}
\end{array}\right),
$$

where $\check{G}^{R, A, K}$ are the retarded, advanced, and Keldysh components, respectively, which are $8 \times 8$ matrices in the Nambu-spin space.

The expression for the charge current $I_{q}$ taking into account the spin-filter effect was derived in Refs. 42 and 50 and reads

$$
\begin{aligned}
I_{q}= & {\left[16 e R_{N}\left(\mathcal{T}^{2}+\mathcal{U}^{2}\right)\right]^{-1} } \\
& \times \int d \epsilon \operatorname{Tr}\left\{\hat{\tau}_{3}\left[\check{\Gamma}^{\check{G}_{R}}(\epsilon) \check{\Gamma}^{\dagger}, \check{G}_{L}(\epsilon)\right]^{K}\right\},
\end{aligned}
$$

where $\mathcal{T}$ and $\mathcal{U}$ are the tunneling spin-independent and spin-dependent matrix elements (for simplicity we neglect their momentum dependence), $\check{\Gamma}=\mathcal{T}+\mathcal{U} \tau_{3} \otimes \sigma_{3}, \quad R_{N}=$ $\left[4 \pi e^{2} N_{L}(0) N_{R}(0)\left(\mathcal{T}^{2}+\mathcal{U}^{2}\right)\right]^{-1}$ is the junction resistance in the normal state, $N_{\mathrm{R}(\mathrm{L})}$ are the density of the states at the Fermi level in the left or right electrode, respectively, and $e$ is the electron charge. In analogy and following the derivation carried out in Ref. 50 one can demonstrate that the heat current $\dot{Q}$ is given by

$$
\begin{aligned}
\dot{Q}= & {\left[16 e^{2} R_{N}\left(\mathcal{T}^{2}+\mathcal{U}^{2}\right)\right]^{-1} } \\
& \times \int d \epsilon \epsilon \operatorname{Tr}\left\{\left[\check{\Gamma} \check{\Gamma}_{R}(\epsilon) \check{\Gamma}^{\dagger}, \check{G}_{L}(\epsilon)\right]^{K}\right\} .
\end{aligned}
$$

The GFs in Eqs. (2) and (3) have the general structure

$$
\begin{gathered}
\check{G}_{\mathrm{R}(\mathrm{L})}^{R(A)}=\hat{g}^{R(A)} \tau_{3}+\hat{f}^{R(A)}\left[\cos (\phi / 2) i \tau_{1} \pm \sin (\phi / 2) i \tau_{2}\right] \\
\check{G}_{\mathrm{R}(\mathrm{L})}^{K}=\left(\check{G}^{R}-\check{G}^{A}\right) \tanh \left(\frac{\epsilon}{2 T_{\mathrm{R}(\mathrm{L})}}\right),
\end{gathered}
$$

where $\tau_{1,2,3}$ are the Pauli matrices in Nambu space, $\hat{g}^{R(A)}$ is the normal and $\hat{f}^{R(A)}$ the anomalous component of the retarded (advanced) GFs. The latter are $2 \times 2$ matrices in the spin space and are determined by solving the quasiclassical equations in the F-S electrodes. Thus, both $I_{q}$ and $\dot{Q}$ are given by Eqs. (2) and (3) after substituting the values of the GFs at the interface. ${ }^{52}$ For simplicity we assume that the thickness of the $\mathrm{S}$ and $\mathrm{F}$ layers $\left(t_{S}, t_{F}\right)$ is smaller than the characteristic length over which the GFs vary. In such a case one can average the quasiclassical equations over the thickness of the F-S bilayer that is now described by an effective exchange field $(h)$ and superconducting order parameter $(\Delta)$ defined by ${ }^{51} h / h_{0}=N_{F}(0) t_{F}\left[N_{S}(0) t_{S}+N_{F}(0) t_{F}\right]^{-1}$ and $\Delta / \Delta_{0}=N_{S}(0) t_{S}\left[N_{S}(0) t_{S}+N_{F}(0) t_{F}\right]^{-1}$, respectively. In the expressions above, $h_{0}$ is the bare exchange field existing in each ferromagnetic layer, $\Delta_{0}$ the bulk superconducting energy gap, and $N_{F, S}(0)$ is the density of states at the Fermi level 
in the $\mathrm{F}$ or $\mathrm{S}$ layer, respectively. The normal and anomalous functions in Eqs. (4) and (5) are given by Ref. 53 (we skip the upper indices $\mathrm{R}$ and $\mathrm{A}$ )

$$
\begin{gathered}
\hat{g}=\frac{g_{+}+g_{-}}{2}+\frac{g_{+}-g_{-}}{2} \sigma_{3} \\
\hat{f}=f_{s}+f_{t} \sigma_{3},
\end{gathered}
$$

where $f_{s}=\left(f_{+}+f_{-}\right) / 2$ is the singlet, and $f_{t}=\left(f_{+}-f_{-}\right) / 2$ is the triplet (with vanishing total spin projection) components of the condensate, and

$$
\begin{aligned}
g_{ \pm}^{R} & =\frac{(\epsilon \pm h)}{\sqrt{(\epsilon \pm h+i \eta)^{2}-\Delta^{2}}} \\
f_{ \pm}^{R} & =\frac{\Delta}{\sqrt{(\epsilon \pm h+i \eta)^{2}-\Delta^{2}}} .
\end{aligned}
$$

The same expressions hold for the advanced GFs if we substitute $i \eta$ by $-i \eta$. The latter parameter describes the inelastic scattering rate within the relaxation time approximation ${ }^{54}$ and it is set $\eta=10^{-5} \Delta_{0}$ throughout the article. The density of the states of the electrodes is given by the real part of $g_{+}^{R}+g_{-}^{R}$. Notice that the order parameter $\Delta$ in Eqs. (8) and (9) has to be calculated self-consistently from the gap equation $\ln \left(\Delta_{0} / \Delta\right)=\int_{0}^{\hbar \omega_{D}} d \varepsilon\left(\varepsilon^{2}+\Delta^{2}\right)^{-1 / 2}\left[f_{+}(\varepsilon)+f_{-}(\varepsilon)\right]$, where $f_{ \pm}(\varepsilon)=\left\{1+\exp \left[\frac{1}{T}\left(\sqrt{\varepsilon^{2}+\Delta^{2}} \mp h\right)\right]\right\}^{-1}$ and $\omega_{D}$ is the Debye frequency of the superconductor. Equations (3)-(9) are used in the next sections in order to analyze the heat transport through a variety of tunneling junctions based on the prototypical example of Fig. 1(a).

\section{RESULTS}

We now use the above derived equations to determine the heat transport through Josephson junctions with spin filters. While the charge current (quasiparticle and Josephson components) in such structures has been analyzed both experimentally (in Al-EuS-Al, ${ }^{43}$ and $\mathrm{NbN}-\mathrm{GdN}-\mathrm{NbN}^{49}$ junctions) and theoretically discussed, ${ }^{42,50}$ heat transport in $\mathrm{S}-\mathrm{I}_{\mathrm{sf}}-\mathrm{S}$ has not be studied so far. In what follows we present the results for the thermal conductance, $\kappa=\dot{Q} / \delta T$, in different structures. $\kappa$ can be obtained from Eq. (3), and in the case of identical electrodes is given by

$$
\kappa=\frac{1}{2 e^{2} R_{N}} \sum_{\alpha= \pm} \int d \epsilon \epsilon\left(\frac{\partial F}{\partial T}\right)\left\{N_{\alpha}^{2}-r M_{\alpha}^{2} \cos \phi\right\},
$$

where $\delta T=T_{L}-T_{R}, \quad(\partial F / \partial T)=-\epsilon /\left[2 T^{2} \cosh ^{2}(\epsilon / 2 T)\right]$, $N_{\alpha}=\left(g_{\alpha}^{R}-g_{\alpha}^{A}\right) / 2, M_{\alpha}=\left(f_{\alpha}^{R}-f_{\alpha}^{A}\right) / 2, r=\frac{\mathcal{T}^{2}-\mathcal{U}^{2}}{\mathcal{T}^{2}+\mathcal{U}^{2}}$, and we have assumed that $\delta T \ll T=\left(T_{R}+T_{L}\right) / 2$. The parameter $r$ is a measure for the spin-filter efficiency $\mathcal{P}=\sqrt{1-r^{2}}$ of the barrier: it is equal to 0 for a $100 \%$ spin-filter efficiency and $r=1$ for a nonmagnetic barrier. The second term in the righthand side of Eq. (10) is the phase-dependent anomalous term, which was obtained for the first time by Maki and Griffin. ${ }^{36}$ According to Eq. (10) the phase-coherent contribution to $\kappa$ is suppressed by increasing the spin-filter efficiency, i.e., by decreasing $r$. The fact that an increasing spin-filter efficiency blocks gradually the phase-dependent contribution to the heat current demonstrates that the latter is due to electron pairs with different spin orientation. As we shall show below, if we allow for triplet pairs with finite total spin projection, the phase-dependent contribution to $\kappa$ does not vanish even if $\mathcal{P}=1$.

\section{A. $\mathbf{S}-\mathbf{I}_{\mathrm{sf}} \mathbf{-}-\mathbf{S}$ junction}

We start our analysis by considering a simple $\mathrm{S}-\mathrm{I}_{\mathrm{Sf}}-\mathrm{S}$ junction. We first assume that there is no exchange field induced in the $\mathrm{S}$ electrodes. This occurs when the coupling between the conducting electrons in the superconducting leads and the magnetic moments localized at the barrier can be neglected, for instance, due the presence of a nonmagnetic oxide between the $\mathrm{I}_{\mathrm{sf}}$ and $\mathrm{S}$ layers. ${ }^{43}$ In such a case, one can set in Eq. (10) $N_{+}=N_{-}$and $M_{+}=M_{-}$. Figures $1(\mathrm{~b})$ and 1(c) show the temperature dependence of $\kappa$ for two values of $\phi$ and different spin-filter efficiencies. Throughout the paper the thermal conductance is shown normalized to that in the normal state, $\kappa^{N}=\mathcal{L}_{0} T / R_{N}$, where $\mathcal{L}_{0}=\pi^{2} k_{B}^{2} / 3 e^{2}$ is the Lorenz number and $k_{B}$ is the Boltzmann constant. If $\phi=0$ the contribution to $\kappa$ from the phase-dependent part is negative, and therefore by decreasing $r$, (i.e., by increasing the efficiency of the spin-filter) the thermal conductance increases [see Fig. 1(b)]. On the contrary, for $\phi=\pi$ the anomalous contribution to $\kappa$ is positive, and the thermal conductance decreases with $r$. With the exception of $r=1$ and $\phi=0$ case, $\kappa$ always shows a maximum at a certain finite temperature $\left(T \approx 0.55 T_{C}\right)$.

We now assume that the density of states of the $S$ layers shows a Zeeman splitting, which acts as an effective exchange field $h$ inside the superconductor in accordance with Eq. (8). This occurs in the case of thin enough $S$ layers and if the contact $\mathrm{I}_{\mathrm{sf}}-\mathrm{S}$ is good enough to allow for a magnetic proximity effect of the $I_{\mathrm{sf}}$ barrier. $^{43,48}$ We note that our model can also describe $\mathrm{SF}-\mathrm{I}_{\mathrm{sf}}-\mathrm{F}-\mathrm{S}$ structures with two thin ferromagnetic films [see Fig. 1(a)]. In Figs. 2(a) and 2(b) we have chosen $h=0.4 \Delta_{0}$ and calculated the temperature dependence of $\kappa$ for $\phi=0$ and $\phi=\pi$, respectively. Due to the presence of the exchange field the superconducting critical temperature of the SF electrodes is reduced by a factor $\sim 0.875$ with respect to the bulk $T_{c}$. The black curves in Figs. 2(a) and 2(b) correspond to a perfect spin filter with $\mathcal{P}=1(r=0)$. According to Eq. (10), in this case, the only contribution to $\kappa$ comes from the quasiparticle channel. As in the zero exchange field case, if $r \neq 0$ the corrections to $\kappa$ from the phase-dependent anomalous term in Eq. (10) are negative for $\phi=0$ and positive for $\phi=\pi$. This explains why for $\phi=0$ the amplitude of the thermal conductance decreases by increasing $r$ [see Fig. 2(a)], whereas for $\phi=\pi$ the thermal conductance increases with $r$ [see Fig. 2(b)].

In Figs. 2(c) and 2(d) we compare the $\kappa(T)$ dependence in the presence and in the absence, respectively, of a spin-filter barrier. Here we set a zero phase difference, $\phi=0$. If the tunneling barrier is nonmagnetic, $r=1$, the transition to the superconducting state leads to a decrease of the thermal conductance as shown in Fig. 2(d). Notably, in this case for any temperature $\kappa$ increases monotonically by enhancing the amplitude of the effective exchange field $h$. By contrast, if the tunneling barrier has a finite spin-filter efficiency $(r=0.5$, which corresponds to $\mathcal{P} \approx 0.88$ ), below the superconducting transition temperature, $T \lesssim T_{c}$, the thermal conductance in- 

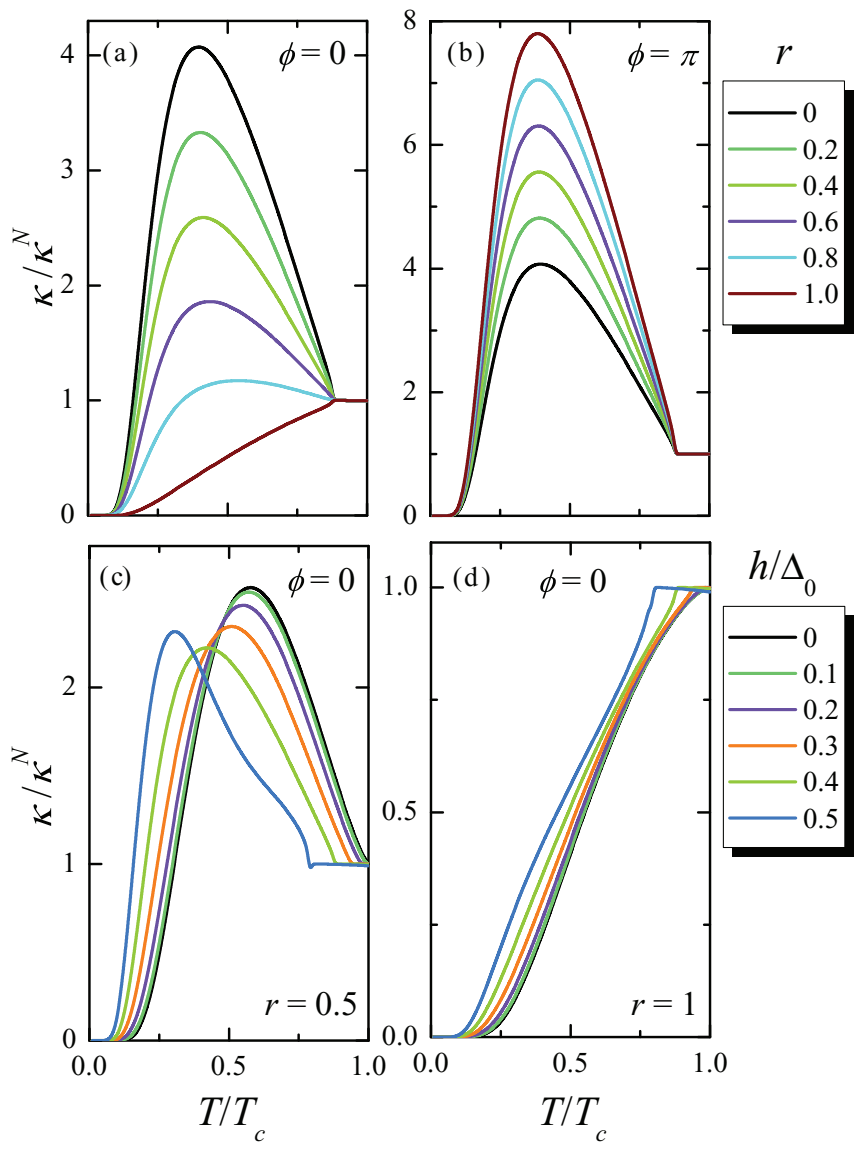

FIG. 2. (Color online) (a) Thermal conductance vs $T$ calculated for several values of $r$ at $\phi=0$. (b) The same quantity as in panel (a) calculated for $\phi=\pi$. In panels (a) and (b) we set $h=0.4 \Delta_{0}$ where $\Delta_{0}$ is the zero-temperature, zero-exchange field superconducting order parameter. (c) Thermal conductance vs $T$ calculated for a few values of $h$ at $\phi=0$ and for finite spin-filter efficiency $(r=0.5)$. (d) The same quantity as in panel (c) calculated in the absence of a spin-filter barrier $(r=1)$.

creases by decreasing the exchange field. By further decreasing the temperature, $\kappa$ shows a maximum, and then decays to zero [see Fig. 2(c)]. The maximum value of $\kappa\left(\kappa_{\max }\right)$ depends nonmonotonically on $h$ : For small enough values of $h, \kappa_{\max }$ decreases by increasing $h$, however for $0.4 \Delta_{0}<h<0.5 \Delta_{0}$ it turns out to increase.

From Eq. (10) it clearly appears that for a spin filter with $100 \%$ efficiency $(r=0)$, the anomalous contribution to $\kappa$ vanishes [i.e., the last term in Eq. (10) is zero] and therefore the heat transport will not depend on the phase difference $\phi$.

\section{B. Triplet Josephson junctions with spin filter}

In order to detect the spin triplet supercurrents, the longrange Josephson effect has been measured in a variety of multilayered ferromagnetic structures ${ }^{24,25,55,56}$ with inhomogeneous magnetic configurations. According to the theoretical prediction, ${ }^{3}$ such inhomogeneity induces the triplet pair correlations with equal spin projection in the ferromagnetic bridge. Here we aim to understand the heat transport through SF hybrid structures containing tunneling barriers. In the context of spin filters, a triplet Josephson current with finite spin
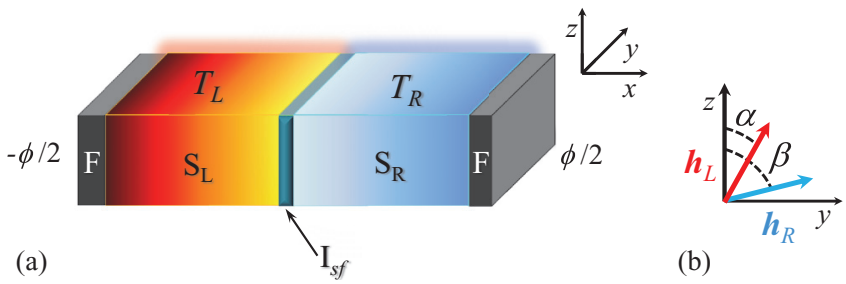

(b)

FIG. 3. (Color online) (a) The triplet FS- $\mathrm{I}_{\mathrm{sf}}-\mathrm{SF}$ Josephson tunnel junction discussed in the text. The two FS bilayer are tunnel coupled by a spin-filter barrier $I_{\text {sf }}$. (b) The exchange fields $\left(h_{\mathrm{L}, \mathrm{R}}\right)$ in the ferromagnetic layers are confined to the $y-z$ plane, and are misaligned by an angle $\alpha$ and $\beta$, respectively, with respect to the $z$ axis. The latter is defined by the magnetization direction of the $I_{\text {sf }}$ barrier.

projection can be observed in junctions as the one sketched in Fig. 1(a), provided that the magnetization of the F layers are noncollinear. However, a more realistic experimental realization of such junction with tunable magnetization is shown in Fig. 3(a), where the outer layers are the ferromagnets. For a good contact between the $\mathrm{S}$ and $\mathrm{F}$ layers and small enough thicknesses this structure is equivalent to the one shown in Fig. 1(a). We set the $z$ axis (spin quantization axis) parallel to the magnetization of the $\mathrm{I}_{\mathrm{sf}}$ layer, and define the angles, $\alpha$ and $\beta$, which describe the direction of magnetization of the left and right ferromagnets, respectively [see Fig. 3(b)]. The generalized expression for the thermal conductance in this case can be derived from Eq. (3) with the help of the technique used in Refs. 42,50. We obtain $\kappa=\kappa_{q p}+\kappa_{\phi}$, where $\kappa_{q p}$ is the contribution from the quasiparticles to thermal transport given by

$$
\begin{aligned}
\kappa_{q p}= & \frac{1}{4 e^{2} R_{N}} \int d \epsilon \epsilon\left(\frac{\partial F}{\partial T}\right)\left\{\left[N_{+}+N_{-}\right]^{2}\right. \\
& \left.+\left[N_{+}-N_{-}\right]^{2} \cos \alpha \cos \beta+r \sin \alpha \sin \beta\left[N_{+}-N_{-}\right]^{2}\right\},
\end{aligned}
$$

and $\kappa_{\phi}$ is the anomalous phase-dependent contribution that can be written in terms of the singlet $\left(f_{s}\right)$ and triplet $\left(f_{t}\right)$ component of the condensate

$$
\begin{aligned}
\kappa_{\phi}= & -\frac{\cos \phi}{4 e^{2} R_{N}} \int d \epsilon \epsilon\left(\frac{\partial F}{\partial T}\right)\left[r M_{s}^{2}\right. \\
& \left.+r M_{t}^{2} \cos \alpha \cos \beta+M_{t}^{2} \sin \alpha \sin \beta\right],
\end{aligned}
$$

where $M_{t} \equiv M_{+}-M_{-}=f_{t}^{R}-f_{t}^{A}$ and $M_{s} \equiv M_{+}+M_{-}=$ $f_{s}^{R}-f_{s}^{A}$. According to the right-hand side of Eq. (12) there are three different phase-dependent contributions to the thermal conductance: The first and the second stem from the singlet and triplet components of the condensate with zero spin projection. As expected, these two terms vanish in the case of an ideal spin-filter barrier $(r=0)$. The last term in the right-hand side of Eq. (12) describes the contribution of the triplet component of the condensate with finite spin projection. It is only finite if the magnetization of the ferromagnetic layers is noncollinear with respect to the magnetization of the barrier $\mathrm{I}_{\mathrm{sf}}$ (i.e., if $\alpha, \beta \neq 0, \pi$ ). Due to this last contribution, the thermal conductance $\kappa$ depends on the superconducting phase $\phi$ even in the case of a $100 \%$ spin-filter efficiency $(r=0)$. In such a case, the measured $\kappa(\phi)$ dependence is a direct manifestation of the triplet component of the condensate in 

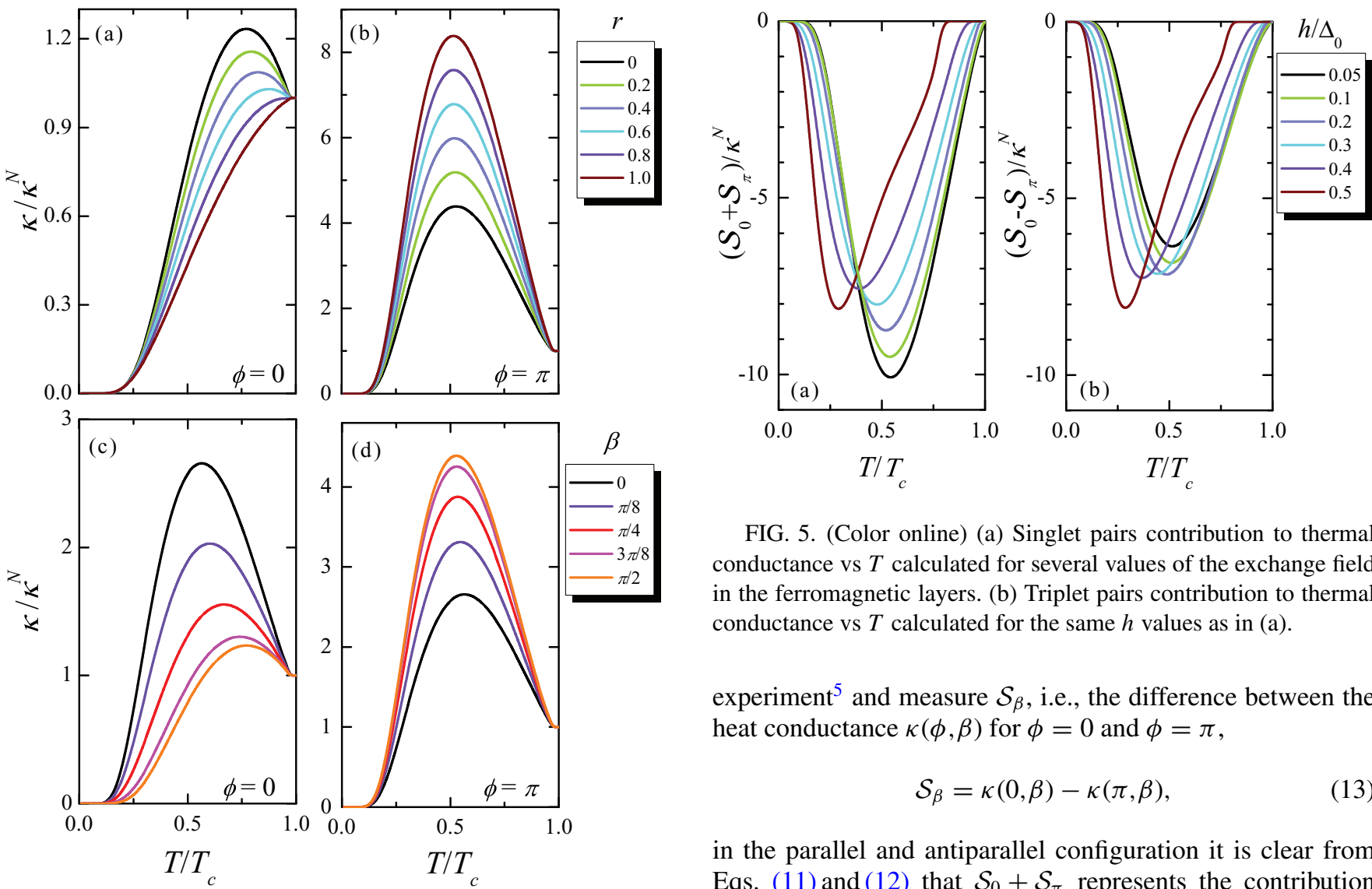

FIG. 5. (Color online) (a) Singlet pairs contribution to thermal conductance vs $T$ calculated for several values of the exchange field in the ferromagnetic layers. (b) Triplet pairs contribution to thermal conductance vs $T$ calculated for the same $h$ values as in (a).

experiment ${ }^{5}$ and measure $\mathcal{S}_{\beta}$, i.e., the difference between the heat conductance $\kappa(\phi, \beta)$ for $\phi=0$ and $\phi=\pi$,

$$
\mathcal{S}_{\beta}=\kappa(0, \beta)-\kappa(\pi, \beta),
$$

in the parallel and antiparallel configuration it is clear from Eqs. (11) and (12) that $\mathcal{S}_{0}+\mathcal{S}_{\pi}$ represents the contribution from singlet pairs

$$
\mathcal{S}_{0}+\mathcal{S}_{\pi}=-\frac{1}{e^{2} R_{N}} \int d \epsilon \epsilon\left(\frac{\partial F}{\partial T}\right) M_{s}^{L} M_{s}^{R},
$$

(a) calculated at $\phi=\pi$. In (a) and (b) we set $\alpha=\beta=\pi / 2$. (c) Thermal conductance vs $T$ calculated for several values of $\beta$ at $\phi=0$. (d) The same quantity as in panel (c) calculated at $\phi=\pi$. In (c) and (d) we set $r=0$ and $\alpha=\pi / 2$. In all the calculations of the figure we assumed $h=0.2 \Delta_{0}$.

analogy to the finite charge supercurrent flowing through a fully efficient spin filter, as recently predicted in Ref. 42 . Again, the phase-dependent contribution $\kappa_{\phi}$ is proportional to $\cos \phi$ [cf. Eq. (10)] and therefore we expect for $\kappa(T)$ a similar behavior as for the $\mathrm{S}-\mathrm{I}_{\mathrm{sf}}-\mathrm{S}$ structure. This is confirmed in Figs. 4(a) and 4(b) where we show the temperature dependence of $\kappa$ for the $\mathrm{F}$ layers having a magnetization parallel to each other but perpendicular to the magnetization of the barrier, i.e., $\alpha=\beta=\pi / 2$. In particular, the thermal conductance can increase considerably with respect to the normal value if $\phi=\pi$. In Figs. 4(c) and 4(d) we show the temperature dependence of $\kappa$ for different angles $\beta$ by setting $\alpha=\pi / 2$. In the $\phi=0$ case maximum values for $\kappa$ are achieved for $\beta=0$, whereas if $\phi=\pi$ the maximum $\kappa$ is observed for $\beta=\pi / 2$.

In principle one can analyze the contributions from the singlet and triplet pairs' density separately by consider the junction of Fig. 3(a) with a nonmagnetic tunneling barrier (i.e., $r=1$ ). We set the magnetization of one of the F layer fixed (e.g., $\alpha=0$ ) and then we switch the other F layer magnetization between a parallel $(\beta=0)$ or antiparallel $(\beta=\pi)$ configuration. If we now perform a phase-biased whereas the difference $\mathcal{S}_{0}-\mathcal{S}_{\pi}$ represents the one from triplet pairs

$$
\mathcal{S}_{0}-\mathcal{S}_{\pi}=-\frac{1}{e^{2} R_{N}} \int d \epsilon \epsilon\left(\frac{\partial F}{\partial T}\right) M_{t}^{L} M_{t}^{R} .
$$
of the temperature for different values of the exchange field. In particular, the maximum contribution from the singlet component is achieved for the lowest values of the exchange field around $T \sim 0.5 T_{c}$, whereas the triplet contribution is maximized by increasing the exchange field value (i.e., in the present case $h=0.5 \Delta_{0}$ ) around $T \sim 0.25 T_{c}$. At large enough exchange fields both contributions tend to be similar. We note that at low temperature the amplitude of the singlet component decreases not monotonically by increasing $h$ whereas that of the triplet contribution turns out to monotonically increase by increasing the exchange field.

\section{Josephson heat valve}

A similar junction as the one shown in Fig. 3(a) (with a nonmagnetic tunneling barrier instead of $I_{s f}$ ) was recently proposed by the authors as a heat valve. ${ }^{41}$ It was shown that the electronic contribution to thermal conductance strongly depends on the relative magnetization angle between the
These two contributions are plotted in Fig. 5 as a function 

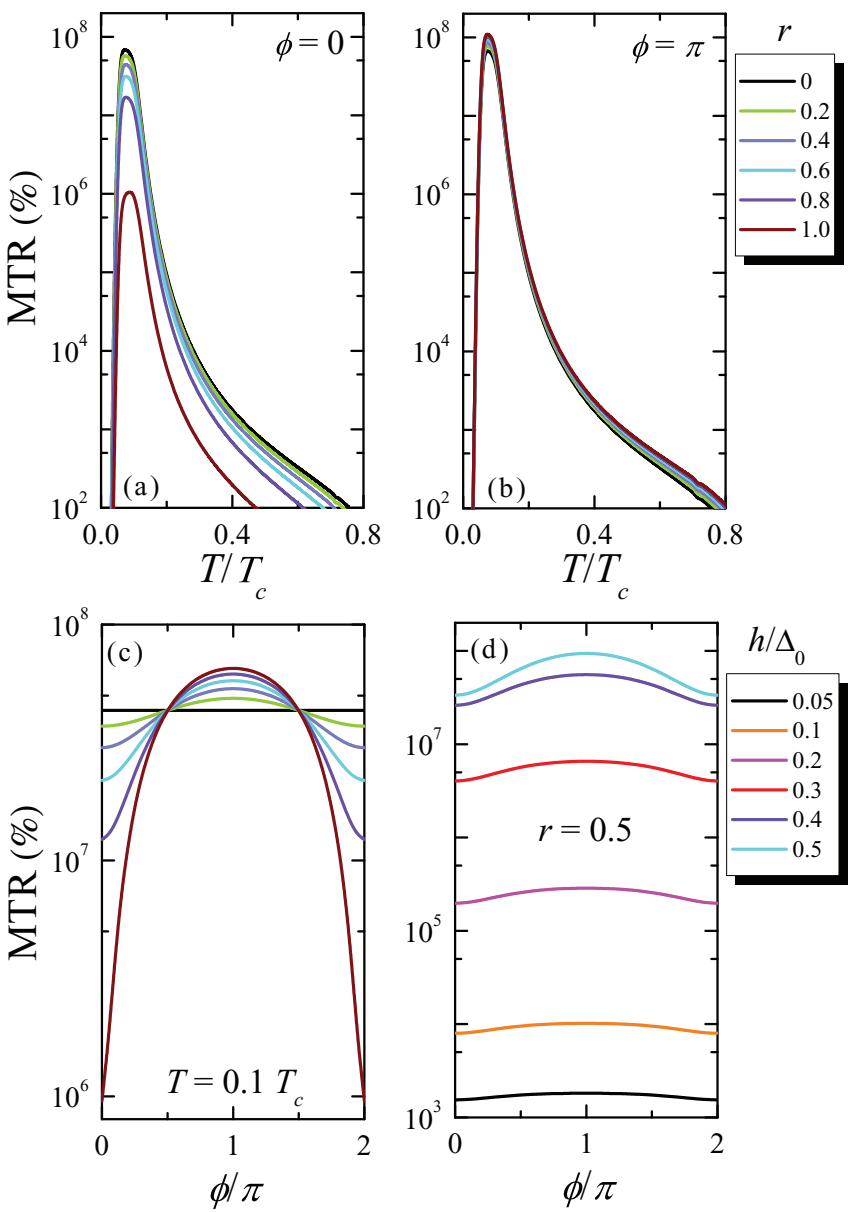

FIG. 6. (Color online) (a) Magnetothermal resistance ratio MTR vs temperature $T$ calculated for several values of $r$ at $\phi=0$ and $h=0.4 \Delta_{0}$. (b) MTR ratio vs $T$ calculated for the same $r$ values as in (a) at $\phi=\pi$ and $h=0.4 \Delta_{0}$. (c) MTR ratio vs phase calculated for the same $r$ values as in (a) at $T=0.1 T_{c}$ and $h=0.4 \Delta_{0}$. (d) MTR ratio vs phase calculated for a few values of the exchange field $h$ at $T=0.1 T_{c}$ and $r=0.5$.

F layers. In particular, values for the magnetothermal resistance (MTR) ratio as large as $10^{5}-10^{7} \%$ has been predicted to occur at low temperature. ${ }^{41}$ The MTR ratio can be defined as

$$
\operatorname{MTR}=\frac{\kappa_{P}-\kappa_{A P}}{\kappa_{A P}},
$$

where $\mathrm{P}$ and AP denote the parallel and antiparallel configuration of the magnetization in the $F$ layers, respectively. In the context of the present paper a natural question arises: How does a spin-filter barrier affect the MTR ratio? The answer to this question can be found in Fig. 6 where we plot the behavior of the MTR as a function of the temperature and the Josephson phase. Figure 6(a) shows that the MTR ratio increases by increasing the spin-filter efficiency for $\phi=0$. In such a case, a $100 \%$ spin-filter efficiency [upper curve in Fig. 6(a)] leads to values of MTR, which are almost two orders of magnitude larger than in the absence of a magnetic barrier $[r=1$, lower curve in Fig. 6(a)]. By contrast, for $\phi=\pi$ the MTR ratio depends only weakly on $r$, and decreases by increasing the spin-filter efficiency. The heat valve effect turns out to be maximized for both phases around $T \sim 0.1 T_{c}$.
The phase dependence of the MTR is plotted in the lower panels of Fig. 6. Figure 6(c) shows this dependence for $T=0.1 T_{c}$ and the same $r$ values as in Figs. 6(a) and 6(b). The MTR ratio is minimized for zero phase difference and reaches its maximum value at $\phi=\pi$. Since we are only considering collinear magnetizations (i.e., either parallel or antiparallel) the phase-dependent contribution to $\kappa$ vanishes if $r=0$ [cf. Eq. (12)], and in turn the MTR ratio does not depend on $\phi$, as shown by the black curve in Fig. 6(c). All curves cross at $\phi=\pi / 2$, which is the phase value separating the two behaviors: If $0 \leqslant \phi<\pi / 2$ the MTR decreases by increasing $r$ while the opposite behavior is achieved for $\pi / 2<\phi \leqslant \pi$. It is worthwhile mentioning that in the parallel configuration the Josephson valve heat conductance is maximized. In contrast, the dc Josephson effect is maximized by the antiparallel configuration. ${ }^{51}$ This means that in the $\mathrm{P}$ configuration the ferromagnetic Josephson junction behaves as an almost ideal electric insulator whereas in the AP one it behaves as an ideal thermal insulator. ${ }^{41}$

Figure 6(d) shows the phase dependence of the MTR ratio calculated for a few different values of $h$ and a moderate spinfilter efficiency $r=0.5$ at $T=0.1 T_{c}$. It clearly appears that the larger the splitting field induced in the $\mathrm{S}$ layers, the larger is the heat valve effect.

\section{SUMMARY}

In summary, we have presented an exhaustive study of the electronic heat transport in $\mathrm{SF}-\mathrm{I}_{\mathrm{sf}}-\mathrm{SF}$ Josephson junctions with magnetic and nonmagnetic $\mathrm{I}_{\mathrm{sf}}$ tunneling barriers. General expressions for the heat current and heat conductance $\kappa$ were derived taking into account the spin-filter efficiency $\mathcal{P}$ of the barrier. It has been shown that $\kappa$ strongly depends on $\mathcal{P}$. For a given value of the exchange field two behaviors have been found: In the case of a zero phase difference between the $\mathrm{SF}$ electrodes an increasing spin-filter efficiency leads to a increase of $\kappa$, whereas the opposite behavior is achieved if $\phi=\pi$. We have also investigated the heat conductance in the case that the magnetizations of the F layers and the spin-filter are noncollinear. We explicitly computed the contributions to $\kappa$ stemming from singlet and triplet pair correlations. Finally, we have analyzed a heat valve based on a F-S- $\mathrm{I}_{\text {sf }}-\mathrm{SF}$ Josephson junction, and demonstrated that for $\pi / 2<\phi \leqslant \pi$ the lowering the spin-filter efficiency of the barrier leads to a sizable enhancement of the magnetothermal resistance ratio.

We finally discuss here some potential applications of the analyzed structures. Ferromagnetic Josephson heat valves can be used whenever a precise tuning and mastering of the temperature is required, for instance, for on-chip heat management as a switchable heat sink. Furthermore, such a valve setup can be useful as well to tune the operation temperature of radiation sensors. ${ }^{27,57}$ In the context of quantum computation ${ }^{58}$ these elements can also be used to influence the behavior and the dynamics of two-level quantum systems through temperature manipulation. Finally, the strong dependence of the Josephson supercurrent on temperature can be exploited for the realization of controllable thermal Josephson junctions of different kinds. ${ }^{27,59-62}$ 


\section{ACKNOWLEDGMENTS}

The work of F.S.B. was supported by the Spanish Ministry of Economy and Competitiveness under Project No. FIS201128851-C02-02. F.S.B. thanks Professor Martin Holthaus and his group for their kind hospitality at the Physics Institute of the Oldenburg University. F.G. acknowledges the FP7 Program No. 228464 "MICROKELVIN", the Italian Ministry of Defense through the PNRM project "Terasuper", and the Marie Curie Initial Training Action (ITN) Q-NET 264034 for partial financial support. *sebastian_bergeret@ehu.es

† giazotto@sns.it

${ }^{1}$ P. M. Tedrow and R. Meservey, Phys. Rep. 238, 173 (1994).

${ }^{2}$ I. Žutić, J. Fabian, and S. Das Sarma, Rev. Mod. Phys. 76, 323 (2004).

${ }^{3}$ F. S. Bergeret, A. F. Volkov, and K. B. Efetov, Rev. Mod. Phys. 77, 1321 (2005).

${ }^{4}$ M. Eschrig, Phys. Today 64, 43 (2011).

${ }^{5}$ F. Giazotto and M. J. Martínez-Pérez, Nature (London) 492, 401 (2012)

${ }^{6}$ R. W. Simmonds, Nature (London) 492, 358 (2012).

${ }^{7}$ M. J. Martínez-Pérez and F. Giazotto, Appl. Phys. Lett. 102, 182602 (2013).

${ }^{8}$ D. Golubev, T. Faivre, and J. P. Pekola, Phys. Rev. B 87, 094522 (2013).

${ }^{9}$ M. Meschke, W. Guichard, and J. P. Pekola, Nature (London) 444, 187 (2006).

${ }^{10}$ E. V. Bezuglyi and V. Vinokur, Phys. Rev. Lett. 91, 137002 (2003).

${ }^{11}$ J. Eom, C.-J. Chien, and V. Chandrasekhar, Phys. Rev. Lett. 81, 437 (1998).

${ }^{12}$ V. Chandrasekhar, Supercond. Sci. Technol. 22, 083001 (2009).

${ }^{13}$ V. V. Ryazanov and V. V. Schmidt, Solid State Commun. 42, 733 (1982).

${ }^{14}$ G. I. Panaitov, V. V. Ryazanov, and V. V. Schmidt, Phys. Lett. 100, 301 (1984).

${ }^{15}$ P. Virtanen and T. T. Heikkilä, Appl. Phys. A 89, 625 (2007).

${ }^{16}$ H. Rabani, F. Taddei, O. Bourgeois, R. Fazio, and F. Giazotto, Phys. Rev. B 78, 012503 (2008).

${ }^{17}$ H. Rabani, F. Taddei, O. Bourgeois, F. Giazotto, and R. Fazio, J. Appl. Phys. 105, 093904 (2009).

${ }^{18}$ T. T. Heikkilä and F. Giazotto, Phys. Rev. B 79, 094514 (2009).

${ }^{19}$ T. Ojanen and A.-P. Jauho, Phys. Rev. Lett. 100, 155902 (2008).

${ }^{20}$ T. Ruokola, T. Ojanen, and A.-P. Jauho, Phys. Rev. B 79, 144306 (2009).

${ }^{21}$ L. M. A. Pascal, H. Courtois, and F. W. J. Hekking, Phys. Rev. B 83, 125113 (2011).

${ }^{22}$ F. S. Bergeret, A. F. Volkov, and K. B. Efetov, Phys. Rev. Lett. 86, 4096 (2001).

${ }^{23}$ R. S. Keizer, S. T. B. Goennenwein, T. M. Klapwijk, G. Miao, G. Xiao, and A. Gupta, Nature (London) 439, 825 (2006).

${ }^{24}$ J. W. A. Robinson, J. D. S. Witt, and M. G. Blamire, Science 329, 59 (2010).

${ }^{25}$ T. S. Khaire, M. A. Khasawneh, W. P. Pratt Jr., and N. O. Birge, Phys. Rev. Lett. 104, 137002 (2010); Carolin Klose et al., ibid. 108, 127002 (2012).

${ }^{26}$ M. S. Anwar, F. Czeschka, M. Hesselberth, M. Porcu, and J. Aarts, Phys. Rev. B 82, 100501 (2010).

${ }^{27}$ F. Giazotto, T. T. Heikkilä, A. Luukanen, A. M. Savin, and J. P. Pekola, Rev. Mod. Phys. 78, 217 (2006).
${ }^{28}$ Y. Dubi and M. Di Ventra, Rev. Mod. Phys. 83, 131 (2011).

${ }^{29}$ J. T. Muhonen, M. Meschke, and J. P. Pekola, Rep. Prog. Phys. 75, 046501 (2012).

${ }^{30}$ G. E. W. Bauer, E. Saitoh, and B. J. van Wees, Nature Mater. 11, 391 (2012).

${ }^{31}$ F. Giazotto, F. Taddei, R. Fazio, and F. Beltram, Appl. Phys. Lett. 80, 3784 (2002).

${ }^{32}$ O. P. Saira, M. Meschke, F. Giazotto, A. M. Savin, M. Möttönen, and J. P. Pekola, Phys. Rev. Lett. 99, 027203 (2007)

${ }^{33}$ J. P. Pekola, F. Giazotto, and O.-P. Saira, Phys. Rev. Lett. 98, 037201 (2007).

${ }^{34}$ F. Giazotto and M. J. Martínez-Pérez, Appl. Phys. Lett. 101, 102601 (2012).

${ }^{35}$ M. J. Martínez-Pérez and F. Giazotto, Appl. Phys. Lett. 102, 092602 (2013).

${ }^{36}$ K. Maki and A. Griffin, Phys. Rev. Lett. 15, 921 (1965).

${ }^{37}$ G. D. Guttman, B. Nathanson, E. Ben-Jacob, and D. J. Bergman, Phys. Rev. B 55, 3849 (1997).

${ }^{38}$ G. D. Guttman, E. Ben-Jacob, and D. J. Bergman, Phys. Rev. B 57, 2717 (1998).

${ }^{39}$ E. Zhao, T. Lofwander, and J. A. Sauls, Phys. Rev. Lett. 91, 077003 (2003).

${ }^{40}$ E. Zhao, T. Löfwander, and J. A. Sauls, Phys. Rev. B 69, 134503 (2004).

${ }^{41}$ F. Giazotto and F. S. Bergeret, App. Phys. Lett. 102, 132603 (2013).

${ }^{42}$ F. S. Bergeret, A. Verso, and A. F. Volkov, Phys. Rev. B 86, 060506 (2012).

${ }^{43}$ X. Hao, J. S. Moodera, and R. Meservey, Phys. Rev. B 42, 8235 (1990).

${ }^{44}$ T. S. Santos, J. S. Moodera, K. V. Raman, E. Negusse, J. Holroyd, J. Dvorak, M. Liberati, Y. U. Idzerda, and E. Arenholz, Phys. Rev. Lett. 101, 147201 (2008).

${ }^{45}$ G.-X. Miao, M. Müller, and J. S. Moodera, Phys. Rev. Lett. 102, 076601 (2009).

${ }^{46}$ B. Li, N. Roschewsky, B. A. Assaf, M. Eich, M. Epstein-Martin, D. Heiman, M. Münzenberg, and J. S. Moodera, Phys. Rev. Lett. 110, 097001 (2013).

${ }^{47}$ F. Giazotto and F. Taddei, Phys. Rev. B 77, 132501 (2008).

${ }^{48}$ T. Tokuyasu, J. A. Sauls, and D. Rainer, Phys. Rev. B 38, 8823 (1988).

${ }^{49}$ K. Senapati, M. G. Blamire, and Z. H. Barber, Nature Mater. 10, 1 (2011).

${ }^{50}$ F. S. Bergeret, A. Verso, and A. F. Volkov, Phys. Rev. B 86, 214516 (2012).

${ }^{51}$ F. S. Bergeret, A. F. Volkov, and K. B. Efetov, Phys. Rev. Lett. 86, 3140 (2001).

${ }^{52}$ A. F. Volkov, Phys. Rev. B 77, 064521 (2008).

${ }^{53}$ F. S. Bergeret, A. F. Volkov, and K. B. Efetov, Phys. Rev. B 64, $134506(2001)$ 
${ }^{54}$ R. C. Dynes, J. P. Garno, G. B. Hertel, and T. P. Orlando, Phys. Rev. Lett. 53, 2437 (1984); J. P. Pekola, T. T. Heikkilä, A. M. Savin, J. T. Flyktman, F. Giazotto, and F. W. J. Hekking, ibid. 92, 056804 (2004); J. P. Pekola, V. F. Maisi, S. Kafanov, N. Chekurov, A. Kemppinen, Y. A. Pashkin, O. P. Saira, M. Möttönen, and J. S. Tsai, ibid. 105, 026803 (2010).

${ }^{55}$ J. W. A. Robinson, G. B. Halasz, A. I. Buzdin, and M. G. Blamire, Phys. Rev. Lett. 104, 207001 (2010).

${ }^{56}$ L. Y. Zhu, Y. Liu, F. S. Bergeret, J. E. Pearson, S. G. E. te Velthuis, S. D. Bader, and J. S. Jiang, Phys. Rev. Lett. 110, 177001 (2013).
${ }^{57}$ F. Giazotto, T. T. Heikkilä, G. P. Pepe, P. Helisto, A. Luukanen, and J. P. Pekola, Appl. Phys. Lett. 92, 162507 (2008).

${ }^{58}$ M. A. Nielsen and I. L. Chuang, Quantum Computation and Quantum Information (Cambridge University Press, Cambridge, 2002).

${ }^{59}$ F. Giazotto and J. P. Pekola, J. Appl. Phys. 97, 023908 (2005).

${ }^{60}$ S. Tirelli, A. M. Savin, C. P. Garcia, J. P. Pekola, F. Beltram, and F. Giazotto, Phys. Rev. Lett. 101, 077004 (2008).

${ }^{61}$ A. M. Savin, J. P. Pekola, J. T. Flyktman, A. Anthore, and F. Giazotto, Appl. Phys. Lett. 84, 4179 (2004).

${ }^{62}$ F. Giazotto, T. T. Heikkilä, F. Taddei, R. Fazio, J. P. Pekola, and F. Beltram, Phys. Rev. Lett. 92, 137001 (2004). 